\title{
Nursing Students' Experiences in Providing Patient Education in the Clinical Setting
}

\author{
Talar Terzian ${ }^{1}$, Jennifer Moradian Watson ${ }^{2} \&$ Shauna Miller $^{1}$ \\ ${ }^{1}$ School of Nursing, California State University, Fresno, USA \\ ${ }^{2}$ Kremen School of Education and Human Development, California State University, Fresno, USA \\ Correspondence: Talar Terzian, School of Nursing, California State University, Fresno, USA.
}

Received: November 30, 2021

Accepted: December 14, 2021

Online Published: December 16, 2021

doi:10.20849/ijsn.v6i4.956

URL: https://doi.org/10.20849/ijsn.v6i4.956

\begin{abstract}
Background: Patient education is associated with reduced hospital readmission rates, lower patient anxiety, and improvement in quality of life. Although nursing students report feeling ill-prepared and less confident in educating their patients in clinical settings, few studies have assessed their perspectives on this topic.
\end{abstract}

Method: This study explores the perceived challenges, obstacles, self-efficacy, and preparedness of undergraduate nursing students in providing patient education by collecting qualitative data through focus group discussions (FGDs).

Results: Five major themes emerged from the FGDs: significance of patient education, nursing process in providing patient education, evolving sense of preparation, evolving sense of confidence, challenges/barriers to providing patient education. While all interviewed nursing students felt that patient education was a vital nursing responsibility, advanced students had more confidence and knowledge regarding this responsibility.

Conclusion: Understanding the nursing student experience in patient education preparedness may aid nursing instructors in better tailoring nursing curricula and support to meet their students' needs.

Keywords: patient education, nursing students, clinical experience

\section{Introduction}

Patient education is a vital nursing responsibility clearly outlined in the scope and standards of nursing (American Nurses Association, 2015) and has significantly evolved in the past six decades (Hoving et al., 2010). Historically, the healthcare provider was viewed as the absolute authority, and patients were required to follow their physician's prescribed regimen. During the past 40 years, patients have been increasingly encouraged to advocate for their health by becoming active participants in their health care, resulting in better patient outcomes. Patient education is associated with increased treatment knowledge of patients (e.g., adverse effects of medications [Apor et al., 2018]), reduced hospital readmission rates, improved quality of life (Rice et al., 2018), and lower patient anxiety levels (Apor et al., 2018; Gökçe \& Arslan, 2019).

Despite the benefits of providing health education to patients, studies have shown the discontent of some patients with nurse-administered health education. Patients have reported being dissatisfied with their nurse's level of knowledge of their condition and allocation of time to discuss their healthcare needs (Albarran et al., 2013; Auyeung et al., 2011; Cooper \& Garrett, 2014). Others reported being confused about their healthcare regimen and not remembering discussing necessary changes to their medications with their healthcare providers (Mackridge et al., 2017). This discontent likely partially stems from the complexity of patient education-patients must process the information provided by their healthcare provider and use it to make well-informed decisions about their healthcare regimen (Graham \& Brookey, 2008). For that reason, nurses must follow the nursing process (Figure 1) and account for learning barriers when teaching patients (Hinkle \& Cheever, 2018). However, while nursing curricula should prepare students to provide effective patient education (American Nurses Association, 2015), this topic may be de-emphasized as nursing instructors are often more concerned with students' practical skills (Abbasi et al., 2018). Accordingly, scarce information exists about how and to what extent nursing school curricula cover patient education, with no information on how often it is covered in nursing programs. While some studies have examined how health literacy is incorporated into nursing school curricula (McCleary-Jones 2012; Sand-Jecklin et al., 2010; Scott, 2016), health literacy is not a primary 
focus of all undergraduate nursing programs (Scott, 2016).

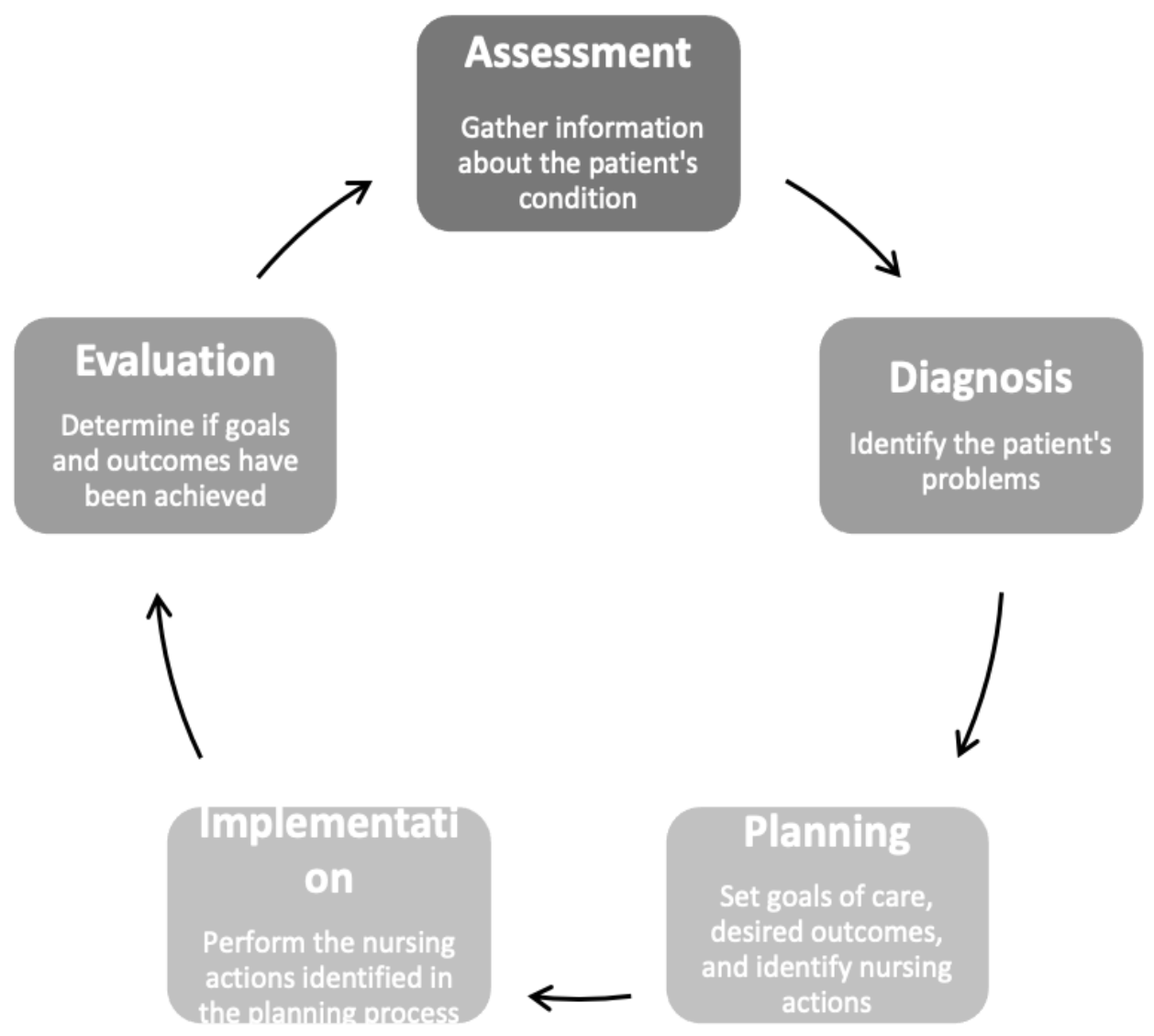

Figure 1. Diagram of the nursing process

Using a qualitative case study design, we explored the perceived challenges, obstacles, self-efficacy, and preparedness of undergraduate nursing students in providing patient education. Uncovering this information will aid nursing instructors in better tailoring nursing curricula to meet the needs of the students in classrooms and clinical settings.

\section{Method}

\subsection{Study Population}

The case study participants consisted of a purposeful sample of nursing students enrolled in the first through fifth semesters (60 students/semester) of the undergraduate Bachelor of Science in Nursing program at a four-year institution in Central California.

\subsection{Qualitative Data Collection}

A case study design and focus group discussions (FGDs) were employed to conduct an in-depth analysis of nursing students' perceived barriers, confidence level, and level of preparedness when providing patient education in the clinical setting (Bhattacharya, 2017). Purposeful sampling was used to recruit participants, as it is a preferred approach for studies seeking a sample of individuals to provide the researcher with rich information about the research problem (Creswell \& Poth, 2018). The study was reviewed and approved by an Institutional Review Board at X, and informed consent was administered to and signed by all students prior to FGD participation.

Students were invited in person by the researcher to participate in five separate FGDs corresponding to their current nursing program semester, which allowed students to ask any clarifying questions, thus encouraging them to participate in the focus groups. Each FGD consisted of 5 to 10 students and were primarily conducted in a neutral space over two weeks. $\mathrm{X}$ is a nursing instructor and moderated the FGDs using a standardized interview protocol, which included a topic guide and a script to ensure that all focus groups received consistent instructions. 
Participants were provided food and drinks during the FGD and received a \$10 Starbucks gift card incentive. The FGDs were recorded and then transcribed through an online service called Transcribe Me. Participating students also completed a brief demographic survey on paper before the start of each FGD.

\subsection{Theoretical Framework and Interview Questions}

The theoretical framework for this study was Benner's (2001) application of the Dreyfus Model of Skill Acquisition, which suggests that as students acquire skills, they go through five different stages of skill acquisition: novice, advanced beginner, competent, proficient, and expert. The interview questions were semi-structured, allowing flexible data collection, as the interviewer was able to ask follow-up and clarification questions based on the participants' responses. To better understand the readiness of undergraduate nursing students to provide patient education through the nursing program, four questions were explored during the FGDs: (1) What are the barriers that nursing students face when providing patient education in the clinical setting?; (2) How prepared do nursing students feel when providing patient education?; (3) How confident do nursing students feel when providing patient education?; and (4) Is there a difference in the barriers, level of confidence, and level of preparation of nursing students in providing patient education as they progress through the nursing program?

\subsubsection{Qualitative Data Analysis}

An open, axial coding system was employed to analyze the qualitative data gathered through the focus groups, allowing the partitioning of data into discrete parts to compare the study participant's responses and interconnect the categories (Strauss \& Corbin, 1998). The moderator/researcher coded the transcripts, extracted the main themes across all participants, and compared participants' perspectives between cohorts.

The researcher used the validation strategies of member checking, peer review, and reflexivity to ensure the study's trustworthiness (Creswell \& Poth, 2018). The recorded interview transcripts were emailed to participants for member checking, and 4 out of the 34 participants replied to the email and confirmed that their answers were correctly transcribed. The peer review involved three researchers with extensive backgrounds in qualitative research methodology and patient education. Each provided comprehensive feedback regarding the research design, data collection methods used, and analysis and interpretation of the data collected. Throughout the data collection and analysis process, the researcher kept a reflexive journal and composed notes about personal experiences supervising nursing students in the clinical setting and teaching them patient education skills. The reflexivity provided the researcher with an opportunity to examine their own biases and assumptions about the findings. The researcher approached data analysis with a clear mind through reflexive journaling and effectively interpreted the participants' experiences.

\section{Results}

\subsection{Participant Characteristics}

A total of 34 participants were interviewed during five separate focus group sessions, with roughly the same percentage representing each semester of the nursing program (Table 1). Most participants were female (94.1\%) and aged between 20 and $29(73.5 \%)$, but the group was racially diverse. Overall, this sample was representative of the students in the program. Participant quotes were edited for length and grammar without altering their content to allow easier result comprehension.

Table 1. Participant and nursing program demographics

\begin{tabular}{lll}
\hline Demographic categories & Participant n (\%) & Undergraduate nursing program n (\%) \\
\hline Gender & & \\
$\quad$ Male & $2(5.9 \%)$ & $72(23.2 \%)$ \\
$\quad$ Female & $32(94.1 \%)$ & $238(76.8 \%)$ \\
Race & & \\
African American & $1(2.9 \%)$ & $3(1 \%)$ \\
American Indian & $\mathrm{NA}$ & $1(0.3 \%)$ \\
Asian & $16(47 \%)$ & $73(24 \%)$ \\
Hispanic & $8(23.5 \%)$ & $109(35.1 \%)$
\end{tabular}




$\begin{array}{lll}\text { Non-Resident Alien } & \text { NA } & 2(0.6 \%) \\ \text { Pacific Islander } & \text { NA } & \text { NA } \\ \text { Two or more Ethnicities } & 2(6 \%) & 11(3.6 \%) \\ \text { Unknown } & \text { NA } & 19(6.1 \%) \\ \text { White } & 7(20.6 \%) & 92(29 \%)\end{array}$

Semester in Nursing School

$\begin{array}{lll}\text { First } & 7(20.6 \%) & 65(21 \%) \\ \text { Second } & 6(17.7 \%) & 60(19.3 \%) \\ \text { Third } & 7(20.6 \%) & 62(20 \%) \\ \text { Fourth } & 5(14.7 \%) & 61(19.7 \%) \\ \text { Fifth } & 9(26.4 \%) & 62(20 \%)\end{array}$

Age Range

$\begin{array}{lll}18-19 & 1(3 \%) & 3(1 \%) \\ 20-29 & 25(73.5 \%) & 274(88.4 \%) \\ 30-39 & 6(17.5 \%) & 25(8.1 \%) \\ 40-49 & 1(3 \%) & 5(1.61 \%) \\ 50-59 & 1(3 \%) & 3(1 \%) \\ \text { Total } & \mathbf{3 4}(\mathbf{1 0 0 \%}) & \mathbf{3 1 0}(\mathbf{1 0 0 \%})\end{array}$

\subsection{Research Themes}

Five main themes were extracted from the FGDs (Table 2): 1) significance of patient education, 2) the nursing process in providing patient education, 3) evolving sense of preparation, 4) evolving sense of confidence, and 5) challenges and barriers to providing patient education.

Table 2. Themes and relevant information

\begin{tabular}{lll}
\hline \multicolumn{1}{c}{ Theme } & \multicolumn{1}{c}{ Category } & \multicolumn{1}{c}{ Code } \\
\hline & Patient advocacy & $\begin{array}{l}\text { Providing competent care, disease prevention and } \\
\text { management, fostering patient independence, caring, } \\
\text { engaging patient and family in plan of care }\end{array}$ \\
$\begin{array}{l}\text { Significance of } \\
\text { patient education }\end{array}$ & $\begin{array}{l}\text { Nurse's role in patient } \\
\text { education } \\
\text { Collaboration of patient } \\
\text { education amongst the } \\
\text { interdisciplinary healthcare } \\
\text { team members }\end{array}$ & $\begin{array}{l}\text { Nurse viewed as mainly responsible for patient } \\
\text { education } \\
\text { Interdisciplinary team members }\end{array}$ \\
\hline $\begin{array}{l}\text { Assessment } \\
\text { The nursing process } \\
\text { in providing patient } \\
\text { education }\end{array}$ & $\begin{array}{l}\text { Diagnosis } \\
\text { Planning }\end{array}$ & $\begin{array}{l}\text { Assessing patient's baseline knowledge, assessing own } \\
\text { knowledge, assessing barriers to learning } \\
\text { Lack of knowledge, gaps in knowledge } \\
\text { Clinical instructor encouragement, research topic, } \\
\text { gather supplies, draw from past experiences, ask } \\
\text { questions }\end{array}$ \\
& $\begin{array}{l}\text { Explanation, skill demonstration, involving family } \\
\text { members, answering questions, repetition }\end{array}$ \\
& Implementation &
\end{tabular}


Evaluation

High level of preparation

Evolving sense of preparation

No preparation

Return-demonstration, teach-back, assessing body language, patient acknowledgment and encouragement

\begin{tabular}{|c|c|c|}
\hline \multirow{2}{*}{$\begin{array}{l}\text { Evolving sense of } \\
\text { preparation }\end{array}$} & High level of preparation & $\begin{array}{l}\text { Assigned patient education, experience, reviewing } \\
\text { patient information in advance, clinical instructor } \\
\text { guidance }\end{array}$ \\
\hline & No preparation & $\begin{array}{l}\text { No assigned patient education lack of experience, lack } \\
\text { of knowledge, comfort level, lack of confidence }\end{array}$ \\
\hline \multirow{2}{*}{$\begin{array}{l}\text { Evolving sense of } \\
\text { confidence }\end{array}$} & Confident & $\begin{array}{l}\text { Experience, exposure to more patients in a particular } \\
\text { setting/unit, relationship with patients, role models, } \\
\text { knowledge level, accepting knowledge limitations, } \\
\text { clinical instructor support }\end{array}$ \\
\hline & Not confident & $\begin{array}{l}\text { Lack of exposure/experience, self-doubt, fear of not } \\
\text { knowing }\end{array}$ \\
\hline \multirow{12}{*}{$\begin{array}{l}\text { Challenges and } \\
\text { barriers to } \\
\text { providing patient } \\
\text { education }\end{array}$} & Lack of experience & $\begin{array}{l}\text { Lack of exposure, unfamiliar with navigating the } \\
\text { healthcare system }\end{array}$ \\
\hline & Lack of knowledge & Unfamiliar with specific topics \\
\hline & Ambiguous student role & Unclear expectations of student role \\
\hline & Prioritization & $\begin{array}{l}\text { Prioritizing skills, time management, patient } \\
\text { availability }\end{array}$ \\
\hline & Language barrier & Patients who do not speak English \\
\hline & Patient's illness acuity & Pain level, level of orientation \\
\hline & Time & $\begin{array}{l}\text { Limited time in the clinical setting, prioritizing other } \\
\text { tasks }\end{array}$ \\
\hline & Anxiety & $\begin{array}{l}\text { Relationship building, fear of the unknown, } \\
\text { acknowledgment }\end{array}$ \\
\hline & $\begin{array}{l}\text { Lack of opportunity/Lack of } \\
\text { support }\end{array}$ & $\begin{array}{l}\text { More practice, more opportunities, no } \\
\text { support/encouragement from nurse preceptor }\end{array}$ \\
\hline & Patient literacy & Patients with limited literacy \\
\hline & Difficult patients & Patients who refuse education, distrusting patients \\
\hline & Patient's culture & $\begin{array}{l}\text { Unfamiliar with patient's culture and healthcare } \\
\text { customs }\end{array}$ \\
\hline
\end{tabular}

Theme 1: Significance of patient education. Nurse's role in patient education. All 34 students noted patient education as being extremely important within a nurse's scope of practice. By providing patient education, the 
students believed that they could establish a trusting relationship with their patients, treat them with respect and dignity, and alleviate their fears. According to one student:

Just imagine somebody who doesn't have any sort of medical background. It can be really scary to be told, "you have this," or to have all these treatments done to you, and you don't know why or what they are or what's going on. So, I think it will put the patient at ease and establish trust.

Patient advocacy. Students felt that providing education makes them an advocate for their patients. Providing patient education enables students to communicate effectively with their patients with clear and concise information, thereby assisting their adherence to prescribed treatment regimens, preventing complications, reducing return hospital visits, fostering decision-making and independence, and promoting preventative care. One student elaborated: "Five minutes of sitting down could really revolutionize their whole outlook on how they treat themselves and how they see their disease."

Collaboration in patient education amongst the interdisciplinary healthcare team members. The students also explained that while all multidisciplinary care team members should provide patient education, a nurses' role in education far outweighs those of other team members - it is one of their primary responsibilities. They stated that nurses spend the most time with the patient and could better assess their comprehension of the information provided and their learning barriers, repeat information as needed, and avoid medical jargon to ensure patient understanding. One student explained:

The doctor briefly goes in, explains it [patient education], but leaves.... It's the nurse's responsibility, since they're at the bedside with the patient, to keep re-educating them or maybe even answering more questions that they didn't get to ask the provider...I definitely think the nurse has a big role in patient education. I'd say most of the responsibility...since they're with the patient for a longer time.

Theme 2: The nursing process in providing patient education. Students described how they use the nursing process (Figure 1) to educate their patients in the clinical setting. First, the students assess both their knowledge and their patient's knowledge (assessment). They consider gaps in the patient's knowledge through observation, reviewing patient information, and asking questions. They also assess the presence of learning-related obstacles, such as language or literacy barriers, high pain levels, diminished readiness to learn, and any cultural beliefs that may impact education. As one student noted: "I took her ethnicity and background into consideration because there are many myths regarding jaundice... and many opportunities for teaching with Hispanics, specifically Mexicans." Second, the students diagnosed the gaps in knowledge as they observed the patient performing a procedure incorrectly or being admitted to the hospital multiple times with the same diagnosis (diagnosis). One student recalled: "The nurse really quickly went over with the patient how to use an incentive spirometer and then left the room to go do something. The patient was just sitting there panicking. She didn't know what she had to do." Third, the students planned for patient education by gathering necessary supplies (e.g., pamphlets, models, illustrations) and reviewing the content to be taught (planning). They also relied on role models (e.g., clinical instructors and nurse preceptors) to clarify any content they were planning on teaching the patient. Fourth, the students implemented patient education by explaining the patient's diagnosis, medications, and diagnostic testing/results, demonstrating a procedure, clarifying any existing misconceptions, and involving family members (implementation). Finally, the students evaluated the effectiveness of the education provided by asking the patient to teach back the content and demonstrate a procedure (evaluation). For this step, students who conducted evaluations reported reading the patients' body language, asking the patients follow-up questions, and making sure to allow time for patients to ask clarifying questions. One student noted how she used the return demonstration method to evaluate whether a postpartum patient was able to set up and clean a breast pump: "So we would literally sit there, and we'd watch her set up the pump. She showed us how to clean out the parts correctly because certain parts needed to be wet, others didn't."

Theme 3: Evolving sense of preparation. As students progressed through the program, they felt more prepared to provide patient education. Just over a quarter of the participants $(26.5 \%$; $9 / 34)$ stated that they felt ready to provide patient education, especially if they had a patient education assignment embedded within their clinical experience. For example, one student commented: "We had to be prepared, so we studied each medication, how to teach the patient, nursing implications, side effects...It took a lot of preparation." In addition, students also felt more prepared when they had prior experience with patient education or the content they were teaching, opportunities to review the patient's chart in advance, and encouraging role models. For example, one of the students explained: "I felt prepared because we covered that topic last semester."

Only approximately $12 \%$ (4/34) of the participants did not feel prepared to provide patient education- these were usually students who had not been previously assigned patient education. The students mentioned that 
being knowledgeable about common topics and diagnoses in a particular unit and further experience and exposure to various patients would significantly enhance their level of preparation. One student stated:

Honestly, when it comes to preparing further, it's just experience. The more meds you run into, the more time you spend learning the various conditions a patient has and how to treat them can increase your level of preparation. It's time on the floor, time reading, time learning. That's about it.

Theme 4: Evolving sense of confidence. Students' confidence in providing patient education increased as they progressed through the curriculum. One fifth semester student elaborated: "I am way more confident, but...I still have way more to go, but I'm still going to try." Students who were prepared to provide patient education felt very confident in doing so. Besides mastering the educational content, they also mentioned having material review opportunities before education implementation, former patient education experience, encouraging patients, good role models in the clinical setting, and effective communication skills contributing to their confidence levels in clinical settings. In addition, the students appreciated the patients who interacted with them and asked them questions. For example, a first semester student stated: "Knowing that your patient trusts you to educate them builds my confidence because they're looking at you and they want you to teach them... It's just knowing that they want to learn from you."

Students who did not feel confident providing patient education attributed their low confidence levels to a lack of experience, fear of not having all the answers, or having an audience, such as family members, listening to the provided patient education. These students acknowledged that more experience, knowledge, and practice would help them build their confidence in the future.

Other students felt varying levels of confidence depending on the situation. For example, their confidence decreased if they perceived the patient to be more knowledgeable than they were. However, if they perceived the patient to be less knowledgeable than they were, their confidence increased. One student stated: "I had some doctors that I was taking care of in obstetrics, and it made me nervous and less confident because I knew that they know so much." The students stated that their confidence levels increased when they had more experience in teaching, prior knowledge on the topic, and research time.

\section{Theme 5: Challenges and barriers to providing patient education.}

Lack of experience, time, and prioritization of other tasks. Some students said that they simply lacked experience in educating patients. The students recognized that their time in the clinical setting was limited. They often had trouble managing their time effectively and prioritized learning to complete other skills over patient education. One student stated: "Because you're in [the clinical setting] for a certain number of hours and you're like, 'Okay. I want to get the best out of this experience.' And so, you want to do the skills." They recognized that their knowledge levels were also often a barrier because they were unfamiliar with certain subjects and were placed in new settings each semester.

Language differences and patient's culture. The language spoken was another barrier that was mentioned. Many patients did not speak English, and the students found it challenging to find an interpreter. Approximately $41 \%(14 / 34)$ of participants said that a language barrier was inhibiting patient education delivery. Nursing students mentioned the difficulties they faced when trying to find an interpreter and being ignorant of certain cultures' healthcare practices. For example, one fourth semester student stated: "I think there's a couple of barriers to patient education. One of them is for sure the language barrier. I feel like there's times where I can't communicate with the patient because of the language barrier." Patients with limited literacy levels or whose cultural practices were unfamiliar to the student also presented as barriers to providing patient education. For example, one fifth semester student stated: "I was trying to ask and gesture to the female patient, and her husband walked out with me and said, "You talk to me." I wasn't sure if I crossed a cultural thing or if I offended them or anything."

Patient's illness acuity. The severity of a patient's illness also presented as an education barrier. Patients who were in pain or had a diminished level of consciousness were not as receptive to education. For example, a second semester student said:

You can't really teach someone when they're in pain... They were too focused on what was hurting them. So, I couldn't really teach them. And then after I gave them their pain medication, they knocked out, so I couldn't really teach them.

Anxiety, lack of knowledge, and ambiguous student role. Student anxiety in a clinical setting was often spurred by difficulty connecting to patients, not being acknowledged by healthcare team members, and feeling uncomfortable with their knowledge level. Of the participants, $18 \%(6 / 34)$ stated they lacked knowledge in 
specific areas, and 6\% (2/34) felt confused about their role as a student nurse. For example, a second semester student stated: "I feel like I know it's going to be a good day when the nurse who I'm with acknowledges me, and that I'm a student. I'm a person that I have a name."

Lack of opportunity/lack of support, and difficult patients. Of the participants, $12 \%$ (4/34) stated they simply did not have as many opportunities to educate patients but would like to have these opportunities. Dealing with difficult patients and families, such as those who tried to micromanage the nurse or patients who were healthcare providers, intimidated the students. They stated that certain patients did not trust their knowledge level because they were student nurses, and even some patients refused to be educated by student nurses. A fifth semester student stated:

When someone in the patient's family is a nurse, they make sure you know that they're a nurse, and they're micromanaging your care. It's very intimidating. Even when you know what you're doing, and you tell them, and they know you know what you're doing.

Lack of support from the nurse preceptor was also another barrier. A third semester student stated:

I would say a challenge has been the interaction I have with my nurse and how willing she is to take me on and teach me ... or she's very closed off toward me, that influences me not to want to educate patients because then I don't want to make the nurse upset, or irritate her, or kind of do things out of her routine. And I think that has been a really big challenge.

Overcoming barriers. The students mentioned that by being proactive, establishing a trusting relationship with their patients, and acknowledging their limitations, they overcame many challenges of delivering patient education. In addition, they found that researching specific subjects further, communicating their objectives with nurses, stepping outside of their comfort zone, and utilizing all available resources helped them overcome many patient education challenges they faced. For example, one student elaborated:

The way to overcome those challenges has been to be honest to myself and my nurse. I have not touched this subject...I was ignorant. However, being able to ask questions, utilize available resources, and follow up in school...I have this patient, so what can I do in the future? So, be honest at that time... I can't learn everything in this shift, but I can prepare for the next patient with that diagnosis.

This quote describes how a student felt that being proactive, acknowledging her own limitations, and utilizing all the available resources in the clinical setting helped her overcome her barriers to providing patient education.

Comparative Analysis Across all Five Cohorts/Semesters. When comparing responses of beginner to advanced students, several similarities became evident. Students in all five semesters recognized the importance of providing patient education within the nurse's scope of practice. They also all weighed the nurse's responsibility to provide patient education far more critical than anyone within the healthcare team. Regardless of their semester of schooling, all students recognized that by providing patient education, they were patient advocates. One student noted:

So, when I think of patient education, I think it's the number one preventative care that you can give someone. You're arming and enabling [patients] to know about their disease or process, and they're able to protect themselves against any future hospital stays... it's also helpful for medical compliance...so important.

However, the barriers and challenges in providing patient education differed according to enrollment semester. They were largely student-centered for first semester students compared with more advanced students. For example, some first semester students did not follow all the steps in the nursing process while providing patients with health education. During the FGDs, these first semester students often missed the evaluation step when asked about completing the patient education process. Although they educated the patient, they did not evaluate whether the education was successful. In addition, during their first semester, students had trouble navigating the patient's chart to gather relevant background information, presenting another barrier to providing patient education. They referenced their lack of knowledge and experience in providing patient education. Also, they did not always know their role as a student nurse, leading to them shying away from educating their patients. For example, one student elaborated:

For me, the biggest challenge is being a first semester student and not really knowing your place in the clinical care setting... knowing what you can and cannot say or what you can and cannot do.

Second semester students identified that their lack of confidence was mainly due to the fear of not knowing all the answers. When students reached their third semester, they could cope with not knowing all the answers and 
learned how to overcome this challenge. One student stated: "So just to feel like you don't have to know everything kind of gives you a little more confidence." In addition, students did not know how to enhance their level of preparation to provide patient education until they reached the third semester. At this stage of the program, they started to recognize that by being more knowledgeable about common topics and diagnoses in a particular unit, they would significantly enhance their level of preparation. One student elaborated:

One thing is to always be aware of your environment... What is common in this population...in this culture? So just be conscientious about what you most often get and the same with the diagnosis, as well as with the patients. What type of patients, what type of diagnoses are common?

For example, this student is aware that each unit in the clinical setting and its patient population is unique. Being aware of the patient population ahead of time enables a student to be more familiar with the common diagnoses encountered in those populations, thus improving their provision of patient education.

Moreover, during their third semester in the nursing program, students started developing the necessary skills to overcome the challenges and barriers they faced in providing patient education in the clinical setting. These skills included: being proactive, establishing a trusting relationship with their patients, acknowledging their limitations, doing more research on specific subjects, communicating their objectives with nurses, stepping outside of their comfort zone, and utilizing all available resources. The students stated that by being proactive, they were able to create more opportunities for themselves, as one student noted, even if it required "Putting yourself in an uncomfortable and unfamiliar situation."

When students reached their fifth and final semester in the nursing program, they were aware of all available resources within the clinical setting to educate their patients, including their interdisciplinary team members. In addition, students were now able to take advantage of the expertise of mentors around them, such as their nurse preceptors and members of the interdisciplinary team. One student stated: "There's just so many resources, so using them and saying, "I don't know, but I can find out."

\section{Discussion}

This study found that all nursing students in a single program, regardless of their semester of enrollment, felt that patient education was a critical, primary part of a nurse's responsibilities and valued their role as patient advocates. All students reported being familiar with the nursing process, and most of them implemented it when providing education for their patients. Some first semester students forgot to include the evaluation step of the nursing process, and students in the earlier stages of schooling were less confident. They reported more student-centered challenges and barriers to providing patient education. By the third semester, nursing students expressed more confidence and knowledge about providing patient education, and these attributes were more commonly reported the further a student was in the program.

Significance of Patient Education. All participants stated that patient education was a critical nursing task and a primary responsibility, more than any other health care professional. By providing patient education, the nurses were acting as patient advocates, echoing the findings of Richard et al. (2018). However, other studies have shown contrasting results. In Choi et al. (2010), nursing students felt that patients did not necessarily view nurses as health educators and subsequently felt unvalued as educators. Nurses in the same study reported that physical routine nursing care was considered a much higher priority than education. In Ghorbani et al. (2014), most nursing students $(63.3 \%)$ noted that health education was not part of their responsibilities. However, these contrasting findings likely result from research settings differences. Choi et al. (2010) and Ghorbani et al. (2014) conducted studies outside the US, where healthcare norms, regulations, policies, and nursing expectations are different, and accordingly, so are nursing students' perspectives.

The Nursing Process in Providing Patient Education. Participants described using the nursing process to educate their patients in the clinical setting. The nursing process is a scientific method used by nurses to ensure the quality of care (Hinkle \& Cheever, 2018), and the use of this process by the participants shows the effectiveness of the curriculum within this program. Implementation of the nursing process is linked with enhanced critical thinking and quality of nursing care (Raziyeh et al., 2018), clinical competence, and better healthcare outcomes for patients (Silva, 2011; Badin et al., 2015; Silva, 2011).

Evolving Sense of Preparation. As students progressed through the program, they felt more prepared to provide patient education. Their sense of preparedness was often dependent on their perceived knowledge level, experience, and actual assignments to offer patient education in the clinical setting. Students who were assigned a patient education experience and were able to spend time studying the material they would teach their patients felt the most prepared. Also, as students encountered more patient education opportunities, they felt more 
prepared to teach. Other similar qualitative studies (Richard et al., 2018) have not reported this evolving sense of preparation in providing patient education, most likely because they did not conduct a comparative analysis of the perspectives of different student cohorts.

Evolving Sense of Confidence. As students progressed through the curriculum, their confidence in providing patient education also increased. Their sense of confidence depended on their perceived sense of preparation, mastery of content, former experience, amount of patient engagement, and role model encouragement. As the students reached their third semester, they started feeling empowered and could cope with not having all the answers to their patients' questions. These findings were somewhat similar to Richard et al.'s (2018) results, where students also attributed their confidence levels to patient engagement and effective communication skills.

Challenges and Barriers to Providing Patient Education. Student nurses identified the following challenges and barriers as they were educating a patient in the clinical setting: lack of experience, lack of knowledge, ambiguous student role, prioritization of other tasks, language barrier, patient's illness acuity, time, anxiety, lack of opportunity/lack of support, patient literacy, difficult patients, and patient's culture. Other studies have also reported similar barriers in providing patient education-lack of support from physicians or program faculty, lack of communication/knowledge or patient education resources, heavy workload/time constraints, and difficulties stemming from patients' literacy, attitude, and readiness to learn (Ghorbani et al. 2014; Badiyepeymaiejahromi et al. 2016; Abbasi et al. 2018)

Implications for nursing instructors. When reflecting on their clinical experiences, nursing students clearly stated that assigned patient education experiences enhanced their sense of preparedness and level of confidence. Therefore, it would be valuable for instructors to prepare students to provide patient education on the most encountered diagnoses each semester. Prior preparation in providing patient education helps boost the confidence and clinical competence of nursing students. This perspective is supported by other studies as well. For example, nursing students who completed a clinical skills course portrayed higher clinical competence and felt more confident with their nursing skills (Park, 2018). In addition, nursing students who completed an educational program about spiritual care also portrayed higher clinical competence in providing spiritual care for their patients (Van Leeuwen et al. 2008).

Students also mentioned that a lack of exposure, experience, and support negatively impacted their sense of preparedness and confidence in providing patient education. Therefore, clinical instructors should encourage patient education opportunities and place students with nurse preceptors willing to provide those experiences for their students. In addition, clinical instructors should try to assign cooperative patients to their students, as students felt more encouraged to provide patient education when their patients were engaged in the education process and appreciated the students' efforts to teach them. Furthermore, clinical instructors should recognize the limitations in their students' knowledge base and allow them research time when a topic or medical diagnosis has not been covered in the curricula.

Clinical instructors should also be aware of the challenges and barriers to the facilitation of patient education enumerated by nursing students. Multiple interviewed students were unclear about their role in the clinical setting during their first nursing school semester. For example, clinical instructors should be as clear as possible early on about the expectations of student nurses in the clinical setting, making sure to cover patient education-related expectations. Students should be exposed to the different cultures and associated healthcare practices common within their geographic region, as unfamiliarity with certain cultures and their healthcare norms were student-identified barriers. Delgado et al. (2013) found that nurses felt more culturally competent and responsive after participating in an educational session. As language was another barrier mentioned by the students, clinical instructors should advise their students about using certified interpreters within each clinical facility. Karliner et al. (2007) discovered that the use of professional medical interpreters results in effective communication amongst healthcare providers and patients, increased satisfaction of care, and positive patient outcomes. Understanding how to utilize these resources will facilitate better communication among student nurses and their patients.

\section{Limitations}

This study has a few limitations. First, the researcher was employed at the study site, potentially limiting the candidness of student feedback. To address this issue, the researcher allowed an opportunity for each participant to speak and ensured that all participant answers remained confidential (Creswell \& Creswell, 2018). Participants seemed comfortable and willing to share their experiences during the FGDs. Second, the case study design focused on one particular 4-year institution within Central California, potentially limiting its generalizability across other programs. However, a thorough description of the research's context was provided, 
potentially aiding other researchers in making inferences about contextual similarities, as suggested by Polit and Beck (2018). Due to space limitations, the FGDs consisting of participants from the second, third, and fifth semester cohorts were conducted in a non-neutral space. However, despite this, students seemed comfortable enough to share their experiences. Fourth, the researcher obtained few responses during the member checking process - while all 34 participants received transcripts via email, only four replied and confirmed the correct transcription of their answers. Fifth, the purposive sampling design may have resulted in recruiting participants that valued patient education. It is possible that not all nursing students understand the significance of patient education, and the findings of the study may not be transferable to all other nursing students. Despite these limitations, the study has notable strengths, including the researcher's familiarity with the program's curriculum, institutional environment, and expectations, enabling a more thorough analysis of the data and development of a practical list of educational considerations for faculty.

\section{Conclusion}

To date, few studies have explored nursing student perspectives on providing patient education. This study uncovered important attitudes and experiences of student nurses in providing patient education in the clinical setting. Educators in similar institutions might transfer some of the findings and benefit from the study's implications to understand student experiences in providing patient education in the clinical setting. This study is an initial step in obtaining a thorough understanding of the students' experiences. This type of in-depth information can spur necessary changes in the curriculum, better supporting the development of delivering exceptional patient education by nursing students. Future studies should focus on how changes in the curriculum impact the students' experiences, especially in cultivating competence in the clinical environment.

\section{Acknowledgements}

The authors would like to thank the nursing students for sharing their experiences and Karoun H. Bagamian and Lindsey Laytner from Bagamian Scientific Consulting for their writing and edition suggestions.

\section{References}

Abbasi, M., Rabiei, L., \& Masoudi, R. (2018). Experience of nursing students about the barriers to patient education: A qualitative study in Iran. Korean Journal of Medical Education, 30(4), 327-337. https://doi.org/10.3946/kjme.2018.107

Albarran, J. W., Jones, I., Lockyer, L., Manns, S., Cox, H., \& Thompson, D. R. (2013). Patients' perspectives on the educational preparation of cardiac nurses. European Journal of Cardiovascular Nursing, 13(5), 451-458. https://doi.org/10.1177/1474515113507166

American Nurses Association. (2015). Nursing: Scope and standards of practice. Silver Spring, MD: Author.

Apor, E., Connell, N., Faricy-Anderson, K., Barth, P., Youssef, R., Fenton, M., ... Mega, A. (2018). Prechemotherapy education: Reducing patient anxiety through nurse-led teaching sessions. Clinical Journal of Oncology Nursing, 22(1), 76-82. https://doi.org/10.1188/18.cjon.76-82

Auyeung, V., Patel, G., Mcrobbie, D., Weinman, J., \& Davies, G. (2011). Information about medicines to cardiac in-patients: Patient satisfaction alongside the role perceptions and practices of doctors, nurses and pharmacists. Patient Education and Counseling, 83(3), 360-366. https://doi.org/10.1016/j.pec.2011.04.028

Badin, M., Garcia, A., \& Toledo, V. (2015). The applicability of the nursing process in caring for psychiatric patients: An integrative review. SMAD. Revista Eletrônica Saúde Mental Álcool E Drogas (Edição Em Português), 11(4), 243-255.

Badiyepeymaiejahromi, Z., Isfahani, S. S., Parandavar, N., \& Rahmanian, A. (2016). Nursing students' perspectives regarding challenges of patient education in clinical settings. Bangladesh Journal of Medical Science, 15(4), 615-620. https://doi.org/10.3329/bjms.v15i4.30719

Bahador, R. S., Nouhi, E., \& Sabzevari, S. (2018). The effect of nursing process training on critical thinking and quality of nursing care. Majallah-i Bālīnī-i Parastārī Va Māmāyyī, 7(3), 202-209.

Benner, P. (2001). From novice to expert: Excellence and power in clinical nursing practice. New Jersey, NJ: Prentice Hall.

Bhattacharya, K. (2017). Fundamentals of qualitative research: A practical guide. New York, NY: Routledge, Taylor \& Francis Group.

Choi, W. H., Hui, G. K., Lee, A. C., \& Chui, M. M. (2010). Student nurses' experiences and challenges in providing health education in Hong Kong. Nurse Education Today, 30(4), 355-359. 
https://doi.org/10.1016/j.nedt.2009.09.005

Cooper, J. M., \& Garrett, T. (2014). Providing medicines information and education to hospital in-patients: Patients' experiences and preferences. Journal of Pharmacy Practice and Research, 44(4), 213-219. https://doi.org/10.1002/jppr.1035

Creswell, J. W., \& Creswell, J. D. (2018). Research design: Qualitative, quantitative, and mixed methods approaches (5th ed.). Los Angeles, CA: Sage.

Creswell, J. W., \& Poth, C. N. (2018). Qualitative inquiry \& research design: Choosing among five approaches (4th ed.). Los Angeles, CA: Sage.

Delgado, D., Ness, S., Ferguson, K., Engstrom, P., Gannon, T., \& Gillett, C. (2013). Cultural competence training for clinical staff: Measuring the effect of a one-hour class on cultural competence. Journal of Transcultural Nursing, 24(2), 204-213.

Ghorbani, R., Soleimani, M., Zeinali, M., \& Davaji, M. (2014). Iranian nurses and nursing students' attitudes on barriers and facilitators to patient education: A survey study. Nurse Education in Practice, 14(5), 551-556. https://doi.org/10.1016/j.nepr.2014.06.003

Gökçe, E., \& Arslan, S. (2019). Possible effect of video and written education on anxiety of patients undergoing coronary angiography. Journal of PeriAnesthesia Nursing, 34(2), 281-288. Retrieved from https://www.sciencedirect.com/science/article/abs/pii/S1089947218302818

Graham, S., \& Brookey, J. (2008). Do patients understand?. The Permanente Journal, 12(3), 67-69. https://doi.org/10.7812/tpp/07-144

Hinkle, J. L., \& Cheever, K. H. (2018). Brunner \& Suddarth's textbook of Medical-surgical nursing (14th ed.). Philadelphia, PA: Wolters Kluwer.

Hoving, C., Visser, A., Mullen, P. D., \& Borne, B. V. (2010). A history of patient education by health professionals in Europe and North America: From authority to shared decision making education. Patient Education and Counseling, 78(3), 275-281.

Karliner, L., Jacobs, E., Chen, A., \& Mutha, S. (2007). Do Professional interpreters improve clinical care for patients with limited English proficiency? A systematic review of the literature. Health Services Research, 42(2), 727-754.

Mackridge, A. J., Rodgers, R., Lee, D., Morecroft, C. W., \& Krska, J. (2017). Cross-sectional survey of patients' need for information and support with medicines after discharge from hospital. International Journal of Pharmacy Practice, 26(5), 433-441. https://doi.org/10.1111/ijpp.12411

McCleary-Jones, V. (2012). Assessing nursing students' knowledge of health literacy. Nurse Educator, 37(5), 214-217. https://doi.org/10.1097/nne.0b013e318262ead3

Park, S. (2018). Effects of an intensive clinical skills course on senior nursing students' self-confidence and clinical competence: A quasi-experimental post-test study. Nurse Education Today, 61, 182-186. https://doi.org/10.1016/j.nedt.2017.11.028

Polit, D. F., \& Beck, C. T. (2018). Essentials of nursing research: Appraising evidence for nursing practice (9th ed.). Philadelphia, PA, PA: Wolters Kluwer.

Rice, H., Say, R., \& Betihavas, V. (2018). The effect of nurse-led education on hospitalisation, readmission, quality of life and cost in adults with heart failure. A systematic review. Patient Education and Counseling, 101(3), 363-374. https://doi.org/10.1016/j.pec.2017.10.002

Richard, E., Evans, T., \& Williams, B. (2018). Nursing students' perceptions of preparation to engage in patient education. Nurse Education in Practice, 28, 1-6. https://doi.org/10.1016/j.nepr.2017.09.008

Sand-Jecklin, K., Murray, B., Summers, B., \& Watson, J. (2010). Educating nursing students about health literacy: From the classroom to the patient bedside. The Online Journal of Issues in Nursing, 15(3), n.p. https://doi.org/10.3912/OJIN.Vol15No03PPT02

Scott, S. A. (2016). Health literacy education in baccalaureate nursing programs in the United States. Nursing Education Perspectives, 37(3), 153-158. https://doi.org/10.1097/01.nep.0000000000000005

Silva, R. (2011). Implementation of the nursing process in a patient with congestive heart failure: Report study. Revista De Enfermagem UFPE on Line, 5(2), 266-272.

Strauss, A. L., \& Corbin, J. M. (1998). Basics of qualitative research: Techniques and procedures for developing 
grounded theory (2nd ed.). Thousand Oaks, CA: Sage.

Van Leeuwen, R., Tiesinga, L., Middel, B., Post, D., \& Jochemsen, H. (2008). The effectiveness of an educational programme for nursing students on developing competence in the provision of spiritual care. Journal of Clinical Nursing, 17(20), 2768-2781.

\section{Copyrights}

Copyright for this article is retained by the author(s), with first publication rights granted to the journal.

This is an open-access article distributed under the terms and conditions of the Creative Commons Attribution license (http://creativecommons.org/licenses/by/4.0/). 